\title{
Understanding Risk: a substitute for Information?
}

\author{
Swati Jha ${ }^{1}$ \\ ${ }^{1}$ Sheffield Teaching Hospitals
}

July 4, 2021

Title Page: Minicommentary

This is a minicommentary on Reid et al. "How common are complications following polypropylene mesh, biological xenograft 3 and native tissue surgery for pelvic organ prolapse? A secondary analysis from the PROSPECT trial".

Title: Understanding Risk: a substitute for Information?

Author : Swati Jha (MD, FRCOG)

Consultant Gynaecologist and Honorary Senior Clinical Lecturer, Sheffield University. Subspecialist in Urogynaecology

Institute : Department of Urogynaecology, Sheffield Teaching Hospitals NHS Foundation Trust

Address : Sheffield Teaching Hospitals, Level 4, Jessop Wing, Tree Root Walk, Sheffield. S10 2SF

Swati.Jha1@nhs.net

$0044(0) 1142268568$

Fax : 01142268165

\section{Contribution:}

SJ: Conceived the article did the literature search and wrote the article

\section{Disclosures}

None relevant to this report

\section{Word Count: 499}

Title: Understanding Risk: a substitute for Information?

The use of mesh for the management of stress urinary incontinence and pelvic organ prolapse (POP) has caused more controversy in modern times than any other intervention. It is estimated that $10 \%$ of women will require surgery for POP over their lifetime. The Olsen paper of 1997 (Olsen et al. Obstet Gynecol 1997; $89[4], 501-506$ ) highlighted the risk of recurrence at approximately $30 \%$ following native tissue vaginal repair which fuelled the need for a more robust treatment. Analogies were drawn between POP and hernias, and it was extrapolated that the success (Lichtensein. Herniorrhaphy 1987; Am.J.Surg, 153, (6) 553-559) of mesh in prevention of hernia recurrence could be applied to the use of mesh for POP too. This was followed by widespread diffusion into clinical practice without robust evidence of efficacy.

The PROSPECT trial was a pragmatic parallel-group multicentre trial of women having surgery for POP comparing native tissue repairs with mesh augmented repairs (inlay and mesh kits) and biological mesh 
(grafts). It found no difference in outcomes at 2 years and all complications were similar in the groups except mesh complications which was 12\% (Glazener et al. Lancet 2017;389: 381-392).

Chronic pain is the commonest cause of referral following mesh surgery and women are choosing to have mesh removed in the hope of symptom improvement. Pain can manifest as dyspareunia, obturator neuralgia, pudendal neuralgia, back pain and nonspecific pain. This study by Reid et al, is a secondary analysis of the data from PROSPECT, and the authors analyse pain and other complications based on the type of repair.

It is interesting that the complications reported in all groups were relatively low and similar in the different groups. As part of this trial, it would be the most experienced surgeons performing cases, but a nuance we must not forget is that unit's enrolling would likely be those where a urogynecologist was the surgeon. The issue of training arises repeatedly in the Cumberlege report (First Do No Harm. https://www.immdsreview.org.uk/downloads/IMMDSReview_Web.pdf.

Accessed 12.06.21) and this study is possibly a reflection of the fact that when undertaken by the specialists' adverse events are low. Pelvic floor surgery as a subspecialty has in the past been dumbed down and was felt to be the domain of the generalist. This may explain some of the problems encountered with the use of mesh, but I would propose that pelvic floor surgery is the remit and prowess of the urogynaecologist.

There is no problem that surgery cannot make worse. This study confirms that all surgeries have a risk of de novo dyspareunia and vaginal pain, but this is much greater with mesh kits when compared to native tissue repair, mesh inlays or grafts. Given that the use of mesh does not improve outcomes however, the added risk of mesh complications is difficult to justify hence the NICE recommendation to use vaginal POP mesh only in the context of research is welcomed by the Urogynaecologists and it is important that lessons learnt from the Cumberlege Report are not forgotten. 